\title{
How Fabrics Dry
}

\section{Nuclear magnetic resonance spectroscopy helps researchers reveal the relationship between the bound and vapor states of water in drying textiles.}

By Marric Stephens

$\square$ ow exactly does a damp piece of fabric dry in air? It might be thought that such an everyday question was answered long ago. But because of difficulties in precisely measuring the distribution of water in a material, physicists have lacked empirical validation for their models. Using a nuclear magnetic resonance (NMR) technique that they previously applied to drying wood (see Focus: Watching Wood Dry), Philippe Coussot and colleagues at Gustave Eiffel University in France have used nuclear magnetic resonance (NMR) spectroscopy to make such measurements [1]. The results could help researchers understand how moisture in exhaled breath changes the effectiveness of face masks and also how to most efficiently dry clothes.

The water in a wet piece of fabric exists in one of two states: it is either bound to the fabric's structure by hydrogen bonds or circulating as vapor in the fabric's voids. Previous models assumed that when a piece of fabric dried, most water left the material by vapor diffusion. The models also assumed certain transfer dynamics between the two states. However, without measurements, researchers could not validate the parameters

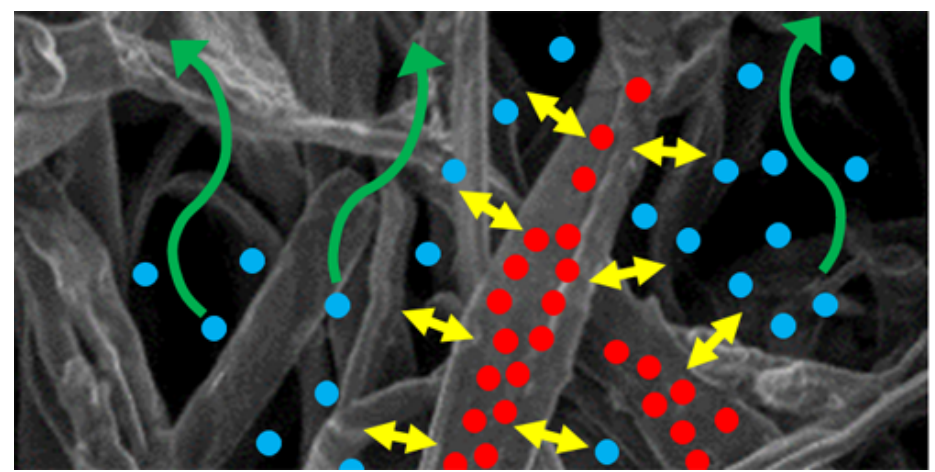

Credit: A. Gossard, J. Lautru, and P. Coussot that they used to make models fit observations.

In their experiments, Coussot and colleagues put samples of wet fabric into open-topped containers and exposed them to flows of dry air. While each piece of fabric dried, the team measured the quantity of bound water throughout the height of the sample via its NMR spin-spin relaxation-time signal. They found that the time evolution of the bound-water profiles was best matched by a model that assumes that the bound and vapor phases achieve equilibrium instantly. The overall drying rate was determined by the dimensions and porosity of the sample and by the properties of the air flow.

Marric Stephens is a Corresponding Editor for Physics based in Bristol, UK.

\section{REFERENCES}

1. X. Ma et al., "Vapor-sorption coupled diffusion in cellulose fiber pile revealed by magnetic resonance imaging," Phys. Rev. Applied 17, 024048 (2022). 